\title{
Efecto coagulante de dos variedades de hoja de coca en muestras de sangre de ratas albinas
}

Clotting effect of two coca leaf varieties on albino rat blood samples

\section{Resumen}

En la presente investigación se evaluó el efecto coagulante de los extractos de dos variedades de hoja de coca: Erytrhoxylum coca lam var. Coca y Erytrhoxylum novogranatense var. Truxillense. El objetivo fue determinar el menor tiempo de coagulación de muestras de sangre de ratas albinas, tratadas con los extractos.

Se seleccionó una muestra de 34 ratas, machos y hembras, a las cuales se le extrajeron $3 \mathrm{ml}$ de sangre a cada una, colocando $1 \mathrm{ml}$ de sangre en tubos que contenían $0.06 \mathrm{ml}$ de suero fisiológico (grupo control), $0.06 \mathrm{ml}$ de extracto de E. coca lam var. Coca (grupo coca), y $0.06 \mathrm{ml}$ de extracto E. novogranatense var. Truxillense (grupo truxillense). Los tiempos de coagulación de las muestras del grupo control fue de $1.42 \mathrm{~min}( \pm 0.31)$, el grupo coca obtuvo $1.38 \mathrm{~min}( \pm 0.26)$ y el grupo truxillense $1.83 \mathrm{~min}( \pm 0.55)$. Los promedios fueron comparados con Anova obteniendo diferencias significativas entre los grupos $(p<0.01)$. Se encontró que el grupo coca produjo el menor tiempo de coagulación que los otros dos grupos. El grupo truxillense mostró un efecto inhibidor de la coagulación comparado al grupo control.

\section{Abstract}

This investigation evaluates the clotting effect of extracts of two varieties of coca leaves: Erytrhoxylum coca var. Coca and novogranatense Erytrhoxylum var. Truxillense. The aim of the study was to determine the shortest time of clotting of the blood samples of the albin rats. 34 males and female rats were selected to have $3 \mathrm{ml}$. of blood of each one. The blood samples were distributed in three probes containing: 0,06 ml. of physiological serum (control group), 0,06 ml. extract of E. coca lam var. coca (coca group), and 0,06 ml. of extract E. novogranatense var. truxillense (truxillense group). The clotting time for the control group was of $1,42( \pm 0.31) \mathrm{min}$, coca group: $1,38( \pm 0.26) \mathrm{min}$, truxillense group 1,83 $( \pm 0.55) \mathrm{min}$. The Anova analysis of the results showed significant differences between groups $(p<0.01)$. The coca group showed the shortest clotting time of all groups. The truxillense group showed an inhibiting clotting effect compared to control group.

\section{Introducción}

Se sabe que los antiguos peruanos utilizaban plantas no solo como alimento, también las empleaban para curar males que los aquejaban. Existen informaciones bibliográficas y folklóricas en la que se da ha conocer el empleo de las plantas para estos fines. Se sabe que las plantas medicinales fueron las bases para diversos tratamientos y muchos fármacos tienen su origen en plantas medicinales.

En la actualidad las plantas son principalmente la fuente de droga para la población mundial. La OMS estima que alrededor de un $80 \%$ de los habitantes del planeta recurren a remedios tradicionales.

La coca tiene diversos usos ejemplo la fabricación de anestésicos o la utilización del extracto de sus hojas como parte de los saborizantes de una bebida gaseosa ${ }^{2,3}$, en la población andina tradicional el uso más conocido que se le da a las hojas de coca es el "chaccheo". Sin embargo, también lo usan como "lubricante" para la interacción social, como medicina, instrumento para adivinar el futuro, diagnostico

\section{Ana María Díaz Sorianoํㅜㄴ Luis Fernando Pérez Vargas ${ }^{1}$, Antonia Castro Rodrí- guez', Sylvia Chein Villacampa', Juan Sánchez Huamaní ${ }^{2}$, Janet Tenorio Estra- da $^{2}$, Ernesto Vilchez Quintana ${ }^{2}$}

Departamento Académico de Estomatología Biosocial

2 Alumnos del segundo año

1,2 Facultad de Odontología. UNMSM

\section{Correspondencia:}

Mg. Ana María Díaz Soriano

Facultad Odontología, UNMSM

Av. Germán Amézaga s/n, Lima, 1 Perú.

e-mail: anamards@yahoo.es

Palabras clave: Hoja de coca, tiempo de coagulación. Variedades de hoja de coca.

Key words: Coca leaf, clotting time, Erythroxylum novogranatense var. Truxillense, Erytrhoxylum coca lam var. Coca. de enfermedades y como asentativo de las comidas. ${ }^{4,2}$

La coca es nativa del Perú y ha sido cultivada desde tiempos muy remotos ${ }^{5}$. Por lo menos desde dos mil años a.c. los habitante del área andina consumían hojas de coca, entonces la antigüedad de la hoja de coca en el Perú es de 4000 años y probablemente más. ${ }^{6,7}$

Los diversos estudios realizados en el Perú acerca de la hoja de coca, lo relacionan generalmente a sus efectos adictivos dados por sus constituyentes 
alcaloides. Pero pocos son los estudios que se refieren a las propiedades benéficas que posee.

Actualmente los estudios realizados sobre la hoja de coca se enfocan en estudiar sus propiedades nutricionales y medicinales. En el aspecto nutricional diversos estudios indican que la hoja de coca es una fuente rica en proteínas, contiene hasta cinco veces el calcio de la leche, ayuda a metabolizar las grasas y los carbohidratos. Además tiene un alto contenido de Selenio, Zinc, Magnesio y vitaminas.

Entre los componentes encontrados en la hoja de coca, además de cocaína y otros alcaloides, tenemos a los taninos, salicilatos y otras sustancias que le proporcionan su benéfica acción medicinal, entre ellas propiedades anestésicas, analgésicas, antidiarreico, evita el soroche (mal de altura), regula la presión arterial, terapéuticas para la gastritis y úlceras, evita la formación de caries dental, ayuda en la coagulación, entre otras. Existen 250 especies conocidas del género Erythroxylum, siendo las especies de mayor presencia en el Perú, Erythroxylum coca Lam. var. Coca y Erythroxylum novogranatense var. Truxillense. Ambas tienen similar composición con diferente concentración, contienen taninos, vitaminas, alcaloides, minerales, carbohidratos, grasas y salicilatos en diferentes proporciones. ${ }^{8}$

En el Perú se han realizado investigaciones sobre las propiedades medicinales de la hoja de coca, entre ellas su propiedad como sustancia coagulante. $^{9}$

Hace más de 200 años se conoce que los taninos presentes en muchos vegetales son pro coagulantes. La actividad pro coagulante del extracto de hoja de coca libre de alcaloides no tiene nada que ver con la cocaína. Este efecto se debe a la presencia de taninos y la hoja de coca lo contiene (falacias de la hoja de coca en la nutrición humana centro de información y educación para la prevención del abuso de drogas).

Por tanto la acción de los taninos encontrados en ambas especies de hoja de coca contribuye a brindarle sus propiedades antiséptica, hemostática $y$ antioxidante.

Estudios anteriores realizados por Espinoza D. y Díaz D. en Tarma y Lima en los años 2004-2005 sustentan el efecto coagulante de Erythroxylum coca Lam. sobre alvéolos pos-exo- doncia en molares permanentes, observándose una disminución notable del tiempo de coagulación de 7,29 a 2,07 minutos.

El objetivo del presente trabajo de investigación fue determinar las diferencias sobre el tiempo de coagulación en muestras obtenidas de ratas albinas de las dos variedades de hoja de coca: Erythroxylum coca Lam, var. Coca y Erythroxylum novogranatense, var. Truxillense.

\section{Material y métodos}

El presente fue un estudio experimental, de controles paralelos no aleatorios, se utilizó 34 ratas albinas de la cepa holtzmann, a las cuales se les extrajo sangre por punción del corazón, con la finalidad de evaluar si el extracto de Erythroxylum coca lam var. Coca aplicado a la muestra de sangre presenta menor tiempo de coagulación que el extracto de Erythroxylum novogranatense var. Truxillense. El empleo de animales se hizo siguiendo las recomendaciones estipuladas en la Declaración de Helsinski de 1975.

Se seleccionaron 17 ratas albinas machos y 17 hembras de la cepa holtzmann, con una edad promedio de 1 año, con un peso corporal entre 200 - 250 gramos. A dichos animales se les mantuvo en un ambiente ventilado, colocando 5 ratas en cada jaula, con alimento balanceado y agua ad-libitum, por un período de 5 días previos al inicio de la experiencia.

\section{Procedimiento}

1. Las ratas fueron anestesiadas con pentabarbitol sódico por vía intraperitoneal.

2. A los tubos $\mathrm{A}$, que fueron el grupo "control", se les adicionó un $1 \mathrm{ml}$ de suero fisiológico.

3. A los tubos B, se le adicionó $1 \mathrm{ml}$. del extracto de Erythroxylum coca lam var. Coca.

4. De igual manera se procedió con los tubos C, se le adicionó $1 \mathrm{ml}$. del extracto de E. Novogranatense var. Truxillense.

5. Se le procedió a la extracción y recolección de la sangre (del corazón) con una jeringa de 10 cc.

6. Los $3 \mathrm{ml}$ de sangre recolectada fue repartida en partes iguales en los tubos A, B y C (1ml/cada tubo).
7. La medición del tiempo de coagulación se realizó de la siguiente manera:

a. Inmediatamente ingresada la sangre al tubo de ensayo se procedió a moverlo para homogeneizar la muestra.

b. Cada tubo se inclinó $45^{\circ}$ aproximadamente cada 30 segundos, para comprobar que la sangre había coagulado.

c. El intervalo de tiempo comprendido entre el ingreso de sangre a la jeringa y el cambio de un estado fluido a otro coloidal se ha interpretado como el "Tiempo de coagulación".

d. El mismo método se aplicó en la medición del tiempo de coagulación en los tres tubos.

\section{Grafico 1. Comparacion de las me- dias del tiempo de coagulacion}

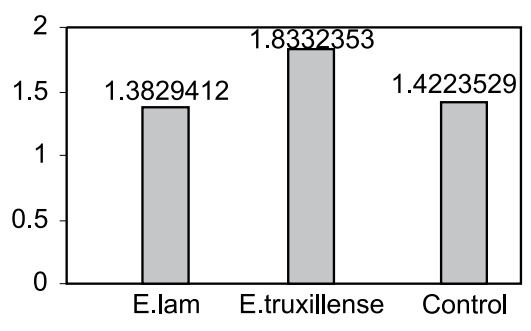

\section{Resultados}

- El menor tiempo de coagulación lo obtuvo el grupo con extracto de E. coca lam var. coca con 1,38 min. $( \pm 0.26)$.

- El mayor tiempo de coagulación lo obtuvo el grupo con extracto de E. novogranatense var. truxillense con una media de $1,83 \mathrm{~min}$. $( \pm 0.55)$.

- Utilizando la prueba estadística ANOVA se muestran diferencias significativas entre grupos $(p<$ 0.01). (Grafico 1).

\section{Discusión}

En la presente investigación se buscó determinar si el extracto de Erythroxylum coca lam var coca presentaba menor tiempo de coagulación que el extracto de Erythroxylum novogranatense var truxillense en muestra de sangre de ratas albinas, lo cual quedó demostrado según los resultados obtenidos, hallándose diferencias significativas (según los análisis estadísticos de ANOVA) con respecto a los tiempos de coagulación de las dos variedades de extracto de coca y el control. 
Los resultados indican que existe menor tiempo de coagulación del extracto de Erythroxylum coca lam var coca con respecto al control, lo cual concuerda con la investigación de Espinoza D y Díaz D (10), quienes utilizaron el mismo extracto en personas y obtuvieron como resultado una disminución en el tiempo de formación del coágulo en alvéolos post exodoncia con respecto a su grupo control.

Al término del estudio se llega a las siguientes conclusiones:

- El mejor efecto coagulante se obtuvo con el extracto de E. coca lam var. coca con $1.38 \mathrm{~min}( \pm 0.26)$ frente a $1.83 \mathrm{~min}( \pm 0.55)$ del grupo con extracto de E. novogranatense var. truxillense.

- El extracto de E. novogranatense var. truxillense mostró un efecto inhibidor de la coagulación con $1.83 \mathrm{~min}$. Frente a $1.4 \mathrm{~min}$ del grupo control.

\section{Referencia bibliográficas}

1. Cabieses F. Aspectos etnológicos de la coca y la cocaína. Cocaína 1980 Seminario Interamericano sobre
Aspectos Médicos y Sociológicos de la Coca y la Cocaína. Lima: Editorial F. R. Jeri; 1980.

2. Cabieses F. Apuntes de medicina tradicional. Lima: A\&B S. A. 1993.

3. Cordero S. Evolución nutricional de la proteína de la hoja de coca. (Trabajo de aptitud académica para optar el título profesional de Químico Farmacéutico). Lima: UNMSM; 2002.

4. Domic Z. Revisión crítica bibliográfica $\mathrm{y}$ consideraciones generales acerca del masticado de coca. Cocaína 1985Seminario Interamericano sobre Aspectos Médicos de la coca y la cocaína. Lima: Editorial F.R. Jeri; 1985.

5. Falero N. y Herrera J. Estudio de la hoja de Erithroxylum cocae Lam. (coca) en úlceras gástricas inducidas en ratas albinas. (Trabajo de aptitud académica para optar el título profesional de Químico Farmacéutico). Lima: UNMSM; 2006.

6. Horna R. La producción de coca en el Perú. Cocaína 1980. Seminario interamericano sobre aspectos médicos y sociológicos de la coca y la cocaína. Lima: editorial F. R. Jeri; 1980.

7. Levano C. A. Estudio fotoquimico de Erithroxylum coca "Hoja de Coca".
Revista de Sanidad de las Fuerzas Policiales 1988;49(2): 130-132.

8. Machado E. El género Erithroxylum en el Perú. Lima; 1968.

9. Mercado R. Aplicaciones higiénicas terapeúticas de la coca. (Trabajo de aptitud académica para optar el título de Médico Cirujano). Lima: UNMSM; 1984.

10. Oyola H. L. Aspectos Bioquímicos del uso de mate de coca. Alma Mater 1993;4:97-100.

11. Rodríguez A. M. Influencia del kerosene doméstico sobre la hemostasia y cicatrización en heridas traumáticas producidas en ratas de experimentación. (Tesis para optar el grado académico de magíster en farmacología con mención en farmacología experimental). Lima: Facultad de farmacia y bioquímica; UNMSM; 2004

12. Venero M. O. Algunas pruebas de laboratorio para apreciar los trastornos de coagulación sanguíneo en perros. (Trabajo de aptitud académica para optar el grado de bachiller en Medicina Veterinaria). Lima: UNMSM; 2006.

Recibido : 27-04-2007

Aceptado para publicación: 25-05-2007

\section{PROYECTOS DE INVESTIGACIÓN APROBADOS - 2007}

- Título: Filtración del enterococcus faecalis en obturaciones retrógradas con MTA comparado con amalgama y ionómero de vidrio Responsable: Pineda Mejía, Martha

- Título: Evaluación del efecto de los microimplantes en el tejido óseo alveolar sometido a distintas fuerzas ortodóncicas

Responsable: Pérez Vargas, Fernando

- Título: Evaluación clínica comparativa de restauraciones clase $\mathrm{V}$ con amalgama fase dispersa y con resina Z350

Responsable: Lahoud Salem, Víctor

- Título: Diagnóstico y tratamiento reversible de trastornos temporomandibulares de pacientes que acuden a la clínica odontológica de la UNMSM. Período 2007 Responsable: Alvarado Menacho, Sergio

- Título: Las células de Sertoli y maduración de los espermatozoides en el epidídimo con ingesta de Maca por ratas jóvenes holtzman

Responsable: Campodónico Reátegui, Carlos

- Título: Mucosa palatina en fetos de 12 a 16 semanas de vida intrauterina Responsable: Sotomayor Tamayo, Justiniano

- Título: Efecto de la vitamina C sobre el movimiento dental ortodóncico en animales de experimentación

Responsable: Rodríguez Alfaro, Miguel 